Pensamiento Crítico Vol. 20 № 1, pp. 145-154

\title{
Tecnología, cambio climático y el sector forestal*
}

\author{
Technology, climate change and forestry
}

[Recepción: Marzo de 2015/ Conformidad: Mayo 2015]

Jazmín Tavera Colonna**

\section{RESUMEN}

Si bien es cierto que el Perú destaca por el potencial natural de su sector forestal, es poco o nada lo que se ha hecho por él. Lo rezagado que se encuentran en manejo tecnológico, los sectores agrícola, forestal y construcción, fuertemente interrelacionados, ha sido totalmente negativo para el sector forestal, así como la alta mecanización de la minería ilegal . Una larga data de un débil marco normativo para un Estado sin capacidad de planificación en un sector que representa una de las mayores riquezas del país, pero con fuertes vínculos intersectoriales, impide convertirlo en uno de alto valor agregado y alta captación de mano de obra en zonas de pobreza extrema. La implementación de actividades de investigación y desarrollo tecnológico en este sector con visión intersectorial es crítico, ya que los beneficios que se deriven de esta implementación permitirán obtener resultados

* Agradezco a mi ex alumna Edith Lucía Cañari Maza por sus aportes de información con respecto a Brasil para la elaboración de este artículo.

** Economista, Magíster en Economía y Maestra en Ciencias. Docente de la Facultad de Ciencias Económicas de la UNMSM. Directora Ejecutiva del Grupo de Estudios y Capacitación. 


\title{
Pensamiento Crítico Vol. 20. NI
}

en un plazo menor. Y es que es un problema que nos urge, el impacto del mal manejo del sector forestal en el cambio climático amenaza con destruir al propio sector forestal. Las mejoras en el marco normativo todavía están pendientes, así como un sistema de información que está en pleno desarrollo, estos avances y el desarrollo en investigación y tecnología del sector con visión integral nos permitirá reconstruir juntos un sector con un gran potencial en valor agregado y combatir con efectividad la pobreza.

Palabras clave: Industria forestal - sostenibilidad - desarrollo tecnológico.

Clasificación JEL: Q 50

\begin{abstract}
While Peru is known for its natural potential for forestry, little or nothing has been done for this sector. The stragglers we are in technology management in agriculture, forestry and construction sectors, strongly interrelated, has been entirely negative for the forestry sector, as the high mechanization of illegal mining. A longstanding weak regulatory framework for a state without planning capacity in a sector that represents one of the greatest riches of the country, but with strong inter sectorial linkages, prevents become one of high value added and high uptake of manpower in areas of extreme poverty. The implementation of research and technological development in this sector with inter sectorial vision is critical, since the benefits arising from this implementation will allow to obtain results in a shorter period. And it is a problem that urges us, the impact of mismanagement of forestry in climate change threatens to destroy the forest sector itself. Improvements in the regulatory framework are still pending, as well as an information system that is in full development, these advances in research and development and technology sector with integrated together allow us to reconstruct a sector with great potential for added value and effectively combat poverty.
\end{abstract}

Keywords: Forestry industry - sustainability - technological development. 


\section{El sector forestal peruano}

De acuerdo al MINAM (2011) el 62\% del territorio nacional son bosques, de los cuales el 59,1\% se encuentran en la Amazonía. Así, el Perú ocupa el undécimo puesto en el mundo en lo que se refiere a superficie de bosques naturales, y el segundo puesto en la región latinoamericana. Paradójicamente, no alcanzamos ni el 1\% de la exportación mundial de productos forestales, siendo la madera el único commodity cuya demanda se mantendrá creciente a largo plazo. En Latinoamérica varios países desarrollan la industria forestal, Brasil, Chile, Argentina y Uruguay. Este desarrollo lo han logrado impulsando plantaciones forestales, que generan una fuente importante de empleo.

Cuadro 1: Empleo, valor de exportaciones y áreas de plantaciones forestales en América Latina

\begin{tabular}{|l|l|l|l|}
\hline \multicolumn{1}{|c|}{ País } & $\begin{array}{c}\text { Empleo en el Sector Forestal } \\
\left(\mathrm{n}^{\circ} \text { de personas) }\right.\end{array}$ & $\begin{array}{c}\text { Valor Exportaciones } \\
\text { (millones USA } \$ \text { ) }\end{array}$ & \multicolumn{1}{|c|}{$\begin{array}{c}\text { Área de plantación forestal } \\
\text { (millones de hás) }\end{array}$} \\
\hline Brasil & $4^{\prime} 000,000$ & 9,500 & 6,5 \\
\hline Chile & 250,000 & 4,000 & 2,1 \\
\hline Argentina & 300,000 & 1,000 & 1,2 \\
\hline Uruguay & 200,000 & 500 & 0,8 \\
\hline Perú & 500 & 395 & 0,04 \\
\hline
\end{tabular}

Fuente: Muñoz Dodero, Fabiola Presente y futuro del sector forestal peruano: el caso de las concesiones y las plantaciones forestales Minagri 2014. Elaboración propia

De los 7,9 millones de $\mathrm{m}^{3}$ de madera extraídos en Perú en el 2012 sólo el 10\% se destinó al uso industrial y comercial. Y en el caso de las exportaciones forestales peruanas, el $80 \%$ de nuestros productos maderables son madera aserrada, madera de parquet y molduras.

La actual Ley Forestal y de Fauna Silvestre No 29763 del 2011 reemplaza a la controversial Ley $N^{\circ} 27308$ del año 2000. Esta nueva ley busca que las concesiones se manejen de manera integral, reconociendo a las plantaciones forestales como cultivos. Crea el Servicio Nacional Forestal y de Fauna Silvestre SERFOR como la autoridad nacional forestal y de fauna silvestre. Para la SPDA (2011) es positiva en lo que respecta a la consolidación de los derechos de comunidades y poblaciones locales, sin embargo, en lo que respecta a la fiscalización se encuentran muchos actores: SERFOR, Gobierno Regional, OSINFOR, OEFA, Ministerio Público, etc., algunos de dudosa responsabilidad sobre el avance de la tala ilegal. Y si bien hay mayor claridad sobre 


\section{Pensamiento Crítico Vol. 20. NoI}

el financiamiento de las actividades forestales, falta aún definir los fondos públicos a largo plazo y el órgano financiador. Son retos aún pendientes en el sector forestal el fortalecer su institucionalidad, generar su desarrollo industrial, mejorar las concesiones forestales en competitividad y control, desarrollar las plantaciones y fomentar aún más la reforestación, así como combatir la deforestación y la tala ilegal.

\section{El Cambio Climático y el Sector Forestal: REDD+ Perú}

Casi el 20\% de las emisiones mundiales de gases de efecto invernadero se deben a la deforestación y degradación de los bosques, ya que un árbol talado libera a la atmósfera todo el CO2 que contenía. En nuestro país la deforestación es la causante del $47 \%$ de nuestras emisiones de gases de efecto invernadero GEI.

En el Perú, la tasa de deforestación para el período 1990-2000 fue menos de 0,1\%, para el 2000-2010 menos de 0,2\%. El Perú sigue una tendencia exactamente inversa a la mundial. Mientras que a nivel mundial se implementan programas de sostenibilidad del bosque, se ejercen controles adecuados para minimizar la tala ilegal, en el Perú, nuestra débil institucionalidad es rebasada por la ilegalidad, haciéndose más crítico por el efecto retroalimentador de la minería ilegal. A esta pérdida de cobertura vegetal e incremento de las emisiones de $\mathrm{CO}^{2}$, hemos de agregar la pérdida de biodiversidad y una sociedad con más conflictos socio ambientales por resolver.

Gráfico 1: Emisiones nacionales de GEI de origen antropogénico por tipo y categoría

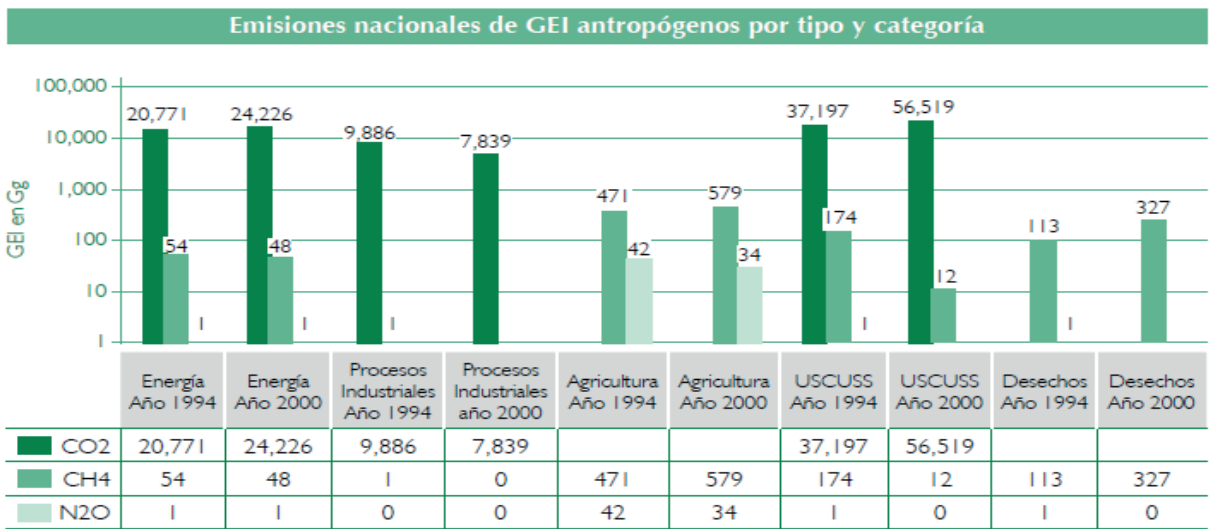

Fuente: Che Piu, Hugo; García, Tania La Situación en REDD en el Perú DAR, GIZ y Red Latinoamericana 2011 
De acuerdo al gráfico № 1 la emisión GEI más importante es el dióxido de carbono y se debe al mal uso del suelo (USCUSS: uso de suelo, cambio del uso del suelo y silvicultura), tendencia que de acuerdo al MINAM se mantendrá por lo menos por 40 años (ver gráfico $\mathrm{N}^{\circ} 2$ ).

Gráfico 2: Emisiones nacionales de GEI de origen antropogénico por categoría

\section{Emisiones nacionales de GEI antropógenos por categoría}

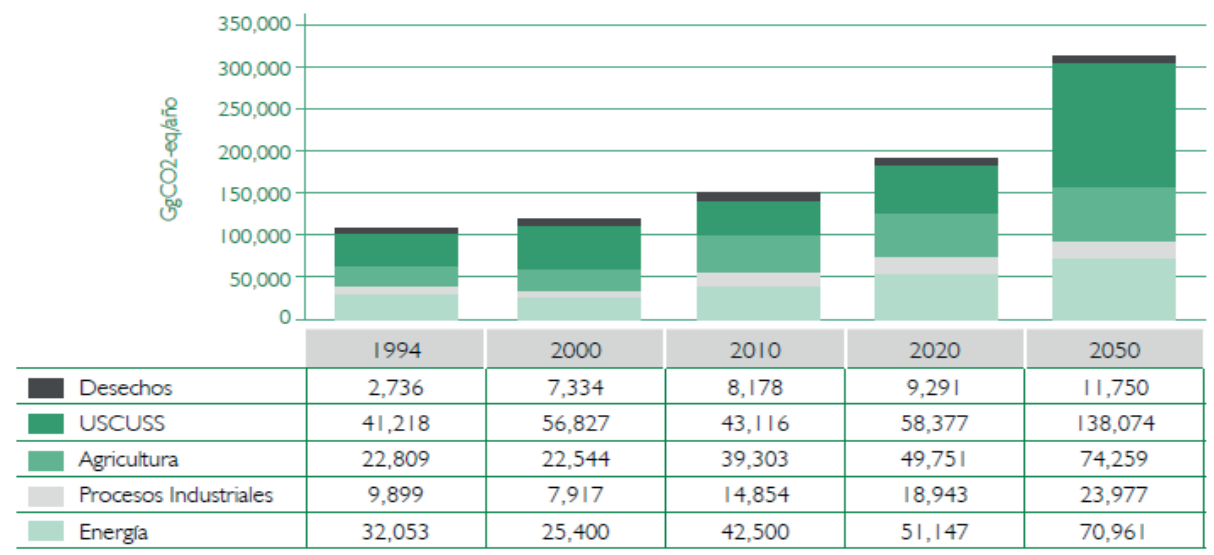

Fuente: Che Piu, Hugo; García, Tania La Situación en REDD en el Perú DAR, GIZ y Red Latinoamericana 2011

Estas emisiones se retroalimentan con la deforestación. Entre las causas de la deforestación tenemos la agricultura y ganadería migratoria, la que se caracteriza por técnicas ancestrales de tumba, roza y quema del bosque asociada a un débil régimen de tenencia de las tierras, los cultivos ilícitos asociados al narcotráfico, una matriz energética que aún tiene a la leña ocupando no menos del $10 \%$ de nuestras fuentes de energía, más el uso agrícola intensivo del suelo para abastecer la agroindustria y la minería ilegal, altamente mecanizada. Sobre esta última sólo en Madre de Dios desde 2008 la deforestación se ha triplicado por la minería ilegal de oro, lo que en este departamento excede de acuerdo a los autores a los efectos combinados de todas las otras causas (Asner et al, 2013). Una causa poco mencionada y tratada en la literatura pero que cobra importancia cada vez más es el desarrollo urbano, el cual trasgrede el equilibrio local al tratarse de asentamientos que reproducen reglas que corresponden a otros 


\section{Pensamiento Crítico Vol. 20. NoI}

entornos y no acorde con los desarrollos técnicos actuales en materia de construcción amigable con el ambiente. Experiencias de ciudades sostenibles en Brasil y Colombia, nos prueban que se puede desarrollar urbes sostenibles minimizando el daño ambiental. En Perú esta iniciativa está siendo trabajada por el INDECI pero bajo la necesidad de minimizar la vulnerabilidad humana ante los desastres naturales.

La Estrategia REDD+ (Reducción de Emisiones por Deforestación y Degradación del Bosque) se orienta a la reducción de emisiones en las actividades forestales generando modelos de negocio para la producción de madera y fijar así el carbono en productos maderables de alta duración. En Perú desde el 2008, Grupo REDD Perú se ha propuesto convertirse en un espacio de interlocución entre las diferentes organizaciones públicas y privadas, basado en la libre participación, transparencia, buena fe y compromiso de sus integrantes. Para ello se han conformado las mesas regionales de Piura, San Martín, Cusco, Madre de Dios, Loreto y Ucayali.

En el 2009 se crea el Programa de Inversión Forestal, y en la Declaración de Tarapoto (2008) se anuncia la reducción de la deforestación neta al 2021 a 0\%. Los objetivos de REDD+ Perú se resumen en 7 puntos, sin embargo, los resultados aún no son los adecuados y su principal limitación es el tratamiento sectorial del problema.

Cuadro 2: Objetivos específicos y resultados del grupo REDD Perú

\begin{tabular}{|c|l|l|}
\hline $\mathrm{N}^{\circ}$ & \multicolumn{1}{|c|}{ Objetivo } & \multicolumn{1}{c|}{ Resultados } \\
\hline 1 & Alcanzar deforestación del 0\% al 2021 & $\begin{array}{l}\text { Creación del Programa Nacional de } \\
\text { Conservación de Bosques Política de } \\
\text { Conservación sin articular con las políticas } \\
\text { sectoriales }\end{array}$ \\
\hline 2 & $\begin{array}{l}\text { Construcción de la posición del país } \\
\text { en las negociaciones internacionales }\end{array}$ & $\begin{array}{l}\text { Proceso aún no ampliamente participativo sólo } \\
\text { informativo } \\
\text { Las articulaciones con los diferentes niveles de } \\
\text { gobierno no se ha dado }\end{array}$ \\
\hline 3 & $\begin{array}{l}\text { Fortalecer procesos de ordenamiento } \\
\text { territorial, zonificación ecológica y } \\
\text { económica, ordenamiento forestal y } \\
\text { catastro }\end{array}$ & $\begin{array}{l}\text { Proyecto de ley en elaboración y sin crearse } \\
\text { aún el catastro forestal }\end{array}$ \\
\hline
\end{tabular}




\begin{tabular}{|c|l|l|}
\hline 4 & $\begin{array}{l}\text { Descentralizar la gestión de bosques } \\
\text { articulado al desarrollo sostenible }\end{array}$ & $\begin{array}{l}\text { Se ha transferido las funciones forestales a 4 } \\
\text { regiones, pero sin recursos financieros }\end{array}$ \\
\hline 5 & $\begin{array}{l}\text { Promover y priorizar la conservación } \\
\text { y manejo sostenible de los bosques } \\
\text { del Perú }\end{array}$ & $\begin{array}{l}\text { Creación del Programa Nacional de } \\
\text { Conservación de Bosques (se empieza a } \\
\text { implementar en 2010) Sin embargo, la } \\
\text { conversión de bosques a otros usos no } \\
\text { sostenibles continúa. }\end{array}$ \\
\hline 6 & $\begin{array}{l}\text { Contar con una institucionalidad } \\
\text { descentralizada que permita viabilizar } \\
\text { iniciativas REDD }\end{array}$ & $\begin{array}{l}\text { Todo se mantiene a nivel de proyectos de Ley } \\
\text { Forestal y Fauna Silvestre y de Provisión de } \\
\text { Servicios Ambientales } \\
\text { La propuesta de institucionalidad para REDD } \\
\text { ha sido presentada en el R-PP }\end{array}$ \\
\hline 7 & $\begin{array}{l}\text { Fortalecer capacidades a nivel } \\
\text { nacional, regional y local }\end{array}$ & $\begin{array}{l}\text { El fortalecimiento más fuerte es a nivel } \\
\text { nacional y muy bajo a nivel local. }\end{array}$ \\
\hline
\end{tabular}

Fuente: Che Piu, Hugo; García, Tania La Situación en REDD en el Perú DAR, GIZ y Red Latinoamericana 2011. Elaboración propia

\section{Tecnología}

La agricultura migratoria es totalmente nociva ya que no permite la fijación del carbono en la madera, la madera es cortada, rozada y quemada, por lo que no sólo no es utilizada, sino que al ser quemada libera inmediatamente el carbono que contenía. La industrialización del sector forestal permitiría convertir esa madera en productos madereros de alto valor agregado, convirtiendo al sector forestal en un sector Carbono Neutral (extraemos lo mismo que producimos de dióxido de carbono). Una industria forestal basada en plantaciones en la Amazonía, costa y sierra es ahora totalmente factible, instalándola por supuesto en zonas deforestadas caracterizadas por la extrema pobreza y escasez de agua. La plantación trae la ventaja de que un bosque en crecimiento a diferencia de uno que ya alcanzó la madurez, permite el secuestro del carbono en una adecuada proporción. Lo que además permitiría absorber mano de obra de áreas de extrema pobreza en la Amazonía, y por supuesto en las áreas deforestadas de costa y sierra, una solución mucho más concreta y efectiva para el problema de la pobreza extrema en nuestro país. En el Perú nunca cumple con el área de reforestación establecida, y en parte se debe a que no reciben una retribución por los servicios ambientales que generan (protección de cuencas hídricas, biodiversidad, suelo), por otra a que los beneficios obtenidos son muy magros. 


\section{Pensamiento Crítico Vol. 20. NoI}

El Perú cuenta desde el 2006, con la Ley de Promoción de la Inversión Privada en Reforestación y Agroforestería, Ley No 28852. Entre los objetivos del Grupo REDD Perú encontramos la necesidad de implementar planes de manejo en el sector tal que asegure su sostenibilidad con la promoción de sistemas agroforestales (único objetivo intersectorial), lo cual puede operar en paralelo a las plantaciones, ya que limita el deterioro del bosque ya existente y se ajusta a pequeños productores en áreas de alta vulnerabilidad ecosistémica.

Por el momento el MINAM se ha concentrado en implementar un adecuado sistema de información del sector, labor del INRENA totalmente desdeñada. MINAM ha suscrito un convenio con el Department of Global Ecology del Carnegie Institution for Science para aplicar tecnologías de sistema de información geográfica SIG y teledetección espacial, lo que ha permitido desde el 2009 la estimación de los stocks de carbono y emisiones para Madre de Dios. Y en un acuerdo múltiple con FAO, entre otros, para que desde el 2011 se desarrolle el Inventario Forestal Nacional que incorpore los valores de la madera, reservas de carbono, emisiones GEI, entre otros. El esfuerzo tiene que ser multisectorial, el cambio climático apremia, y el primer sector afectado será el forestal (Geng, 2006; Vergara, 2008).

En Brasil a pesar de lo agresiva que es su industria de soja, palma aceitera y caña de azúcar (biocombustible), para los mismos períodos estimados para Perú, observó de acuerdo a la FAO, tasas de deforestación menores a las peruanas, aunque con un leve crecimiento (mucho menor que en el caso peruano), 1990- 2000 0,045\%, y en el período 2000-2010 0,048. Para ello el gobierno brasileño, de acuerdo al SIAFI, invirtió para el 2012 en I+D 10\% en el sector agrícola y 6\% en sector industrial. Si el sector agrícola es más productivo, menos área tendrá que ocupar.

\section{Conclusiones}

Con un crecimiento del sector y su consecuente industrialización, los caminos llevarán más población a estas áreas, los que se dedicarán a la industria maderera y a la agricultura y ganadería. Este proceso no se podrá impedir, sin embargo, si se implementa con las nuevas tecnologías ambientalmente amigables estas poblaciones reducirán su impacto en las urbes y en sus actividades rurales, reduciendo la pérdida de cobertura vegetal. La investigación y la tecnología cumplen un rol importante por lo que se debe incentivar su desarrollo e implementación en el sector forestal y los sectores que se encuentran directamente integrados a él. 


\section{Jazmín Tavera Colonna}

Estas mejoras tecnológicas, de una tecnología ya existente, deben acompañarse con la preparación de cuadros de capital humano de calidad que permitan la modernización del sector desde una visión integral, la agricultura y la construcción urbana deben sumarse a este esfuerzo. Así mismo, mejorar nuestra institucionalidad que aún es débil, y engorrosa, y lo más flagrante, aún no incorpora la industrialización del sector, aunque declara pero aún no ordena con claridad la modalidad de plantaciones, lo que además de reducir las emisiones de carbono, elevar productividad y reducir la pobreza, entre algunos de sus beneficios directos, nos beneficiará en la reducción de la informalidad, difícil de combatir por un estado débil y corroído por la corrupción.

\section{Referencias bibliográficas}

Asner, Gregory; Llactayo, William; Tupayachi, Raúl y Ráez Luna, Ernesto (2013) "Elevated rates of gold mining in the Amazon revealed through high-resolution monitoring" Proceedings of the National Academy of Sciences of United States of America en http://www.pnas.org/content/110/46/18454.abstract

Calvo Buendía, Eduardo (2010) "Guía metodológica para la adaptación a los impactos del cambio climático en las ciudades y opciones de mitigación de emisiones de gases de efecto invernadero". Foro Ciudades para la Vida.

Che Piu, Hugo; García, Tania (2011) "La Situación en REDD en el Perú" DAR, GIZ y Red Latinoamericana.

Geng Torres, Luis (2006) "El Futuro del Perú ante el cambio climático" CONAM.

MINAM (2010) "Mapa del Patrimonio Forestal Nacional".

MINAM (2011) "Readiness Preparation Proposal (R-PP)" Versión Final.

MINAM (2012); "Balance en relación a la gestión del cambio climático en el País". Documento de trabajo.

Muñoz Dodero, Fabiola (2014) "Presente y Futuro del Sector Forestal Peruano" MINAGRI en www.bcrp.gob.pe/docs/Publicaciones. 


\section{Pensamiento Crítico Vol. 20. Nº}

Sociedad Peruana de Derecho Ambiental SPDA (2011) "Entendiendo la Ley Forestal y de Fauna Silvestre (Ley 29763)".

El Peruano (2000) "Ley Forestal y de Fauna Silvestre Ley N²7308”.

El Peruano (2006) "Ley De Promoción de La Inversión Privada en Reforestación y Agroforestería Ley No 28852".

El Peruano (2011) “Ley Forestal y de Fauna Silvestre Ley N²9763”.

Vergara, Walter (2008) "Los Andes en Peligro, Impactos inducidos por cambio climático en la cordillera" Banco Mundial. 